\title{
The Effect of Microsurgical Varicocelectomy on Semen Parameters in Men with Non-Obstructive Azoospermia
}

\author{
Mustafa Kiraça Nuri Deniz $^{\mathrm{a}} \quad$ Hasan Birib \\ aDepartment of Urology, Koru Hospital; ${ }^{\mathrm{b}}$ Department of Urology, Gazi University, Ankara,Turkey
}

\section{Key Words}

Azoospermia • Microsurgery • Motile sperm • Varicocele

\begin{abstract}
Introduction: The aim of this study was to evaluate the effect of microsurgical subinguinal varicocelectomy on semen parameters in azoospermic men with clinical varicocele and to determine the predictive parameters of postoperative improvement. Methods: Twenty-three men with non-obstructive complete azoospermia and varicocele underwent subinguinal open microsurgical varicocele repair. The outcome was assessed in terms of improvement in semen parameters after surgical repair for varicocelectomy. Results: Bilateral varicocelectomy was performed on 15 patients and unilateral (left) varicocelectomy was performed on 8 patients. In the post-operative period, of the 23 patients, 7 (30.4\%) had motile sperm in the ejaculate. The mean sperm concentration of these patients was $1.34 \pm 2.6 \times 10^{6} / \mathrm{ml}$ and the mean total sperm motility was $37.5 \pm 15.5 \%$. Conclusion: Infertile men with non-obstructive azospermia can have improvement in semen analysis after subinguinal microsurgical repair of varicoceles. Motile sperm in ejaculate were detected after microsurgical varicocele repair.
\end{abstract}

Copyright $\odot 2012$ S. Karger AG, Basel

\section{KARGER}

Fax +4161306 1234

E-Mail karger@karger.ch

www.karger.com
(C) 2012 S. Karger AG, Basel

1015-9770/12/0063-0136\$26.00/0

Accessible online at:

www.karger.com/cur

\section{Introduction}

Varicocele is a common problem in infertile males, appearing in 10-20\% of the general male population and in $35-40 \%$ of men with primary infertility $[1,2]$. With recent advances in diagnostic techniques, varicocele is frequently reported in subfertile and infertile males. Varicocele is believed to cause progressive deterioration of spermatogenesis by impaired drainage or pooling of blood around the testis, leading to increased scrotal temperature [3]. Azoospermia in association with a varicocele is estimated to range between approximately $4-14 \%$ $[4,5]$. Recent studies have demonstrated improvements in semen parameters after varicocele repair in men with azoospermia [5-7]. The primary benefit of varicocelectomy in men with non-obstructive azoospermia is the possibility of producing motile sperm in the ejaculate. Varicocele repairs in azoospermic men have also increased the success rates of assisted reproductive techniques such as intracytoplasmic sperm injection (ICSI) by providing motile sperm from fresh ejaculate or for testicular sperm extraction (TESE) [5, 8, 9]. Some studies report that varicocele repair using microsurgical technique in patients with azoospermia has resulted in approximately $40 \%$ improvement of semen parameters and the appearance of motile sperm in the ejaculate $[10,11]$.

Mustafa Kiraç, MD

Umit mah. Yeni Çă̆ın Sitesi

A Blok No 38, Yenimahalle

Ankara (Turkey)

E-Mail mkirac@gmail.com 
Thus, the aim of our study was to evaluate the effect of microsurgical subinguinal varicocelectomy on semen parameters in azoospermic men with varicocele and to determine the predictive parameters and motile sperm in postoperative improved ejaculate.

\section{Materials and Methods}

Between October 2006 and February 2011, 23 men with nonobstructive azoospermia and clinical varicocele presenting with primary infertility underwent microsurgical varicocele repair at the Urology Departments of Akay Hospital, Gazi University and Koru Hospital, and were included in this study. A detailed informed consent was obtained from all patients. At least 1 year duration of infertility, defined as failure to establish a pregnancy in 12 months with unprotected intercourse, was required for study group inclusion.

All patients underwent a standard, diagnostic infertility evaluation. A detailed history and complete physical examination was performed by the same physician. Testicular ultrasonography was performed on all patients. Testis volume was assessed by physical examination and testicular ultrasonography. Testicular atrophy was defined as any testis with a volume less than $15 \mathrm{~cm}^{3}$ or a testis $25 \%$ or more smaller (volume/volume) than its controlateral mate [8]. A varicocele identified at scrotal examination, performed with the patient in the standing position and during Valsalva's maneuver, was classified as grade I (palpable only during Valsalva's maneuver), grade II (palpable without Valsalva maneuver) and grade III (visible without need for palpation) [12]. All patients had normal 46, XY karyotypes and no Y chromosome micro-deletions. Hormone analyses including serum testosterone and follicle-stimulating hormone ( $\mathrm{FSH})$ were performed on all patients.

Scrotal color doppler ultrasound was used to confirm the presence of varicocele, to determine the presence of a subclinical varicocele and to evaluate testicular sizes. We used scrotal color doppler ultrasound (Logic 9 with 7.5/10 MHz linear transducer) to determine the varicocele. A varicocele was considered to be present by sonography if two or more veins could be identified, with at least one vein having a diameter of $3.0 \mathrm{~mm}$ or greater.

At least 2 semen analyses were obtained in all patients by masturbation after 2-5 days abstinence. A minimum interval of 2 weeks separated the 2 sperm analyses.

Semen specimens were collected and evaluated according to WHO criteria [13]. Only men with complete azoozpermia (pellet negative) were included in the study. Azoospermia was diagnosed by laboratory findings and was confirmed by the complete absence of sperm from the ejaculate. Complete azoospermia was confirmed by the absence of sperm in the centrifuged semen samples. For pellet analysis, the semen samples were centrifuged for 25 minutes at 3,000 rpm. All patients were fructose positive. Ejaculate volumes for all patients were normal $(>2 \mathrm{ml})$.

For all men in this study, varicocele repair, using a microsurgical subinguinal approach, artery and lymphatic sparing technique was performed [14]. In this technique, through a small transverse skin incision overlying the external inguinal ring, the spermatic cord was grasped with a Babcock clamp. The spermatic cord was then delivered through the incision. The gubernacular veins
Table 1. General characteristics of azoospermic patients

\begin{tabular}{lll}
\hline & $\mathrm{N}(\%)$ & Age (range) \\
\hline $\begin{array}{l}\text { Patient } \\
\text { Varicocele }\end{array}$ & $23(100)$ & $31.7(24-41)$ \\
$\quad$ & & \\
$\quad$ Grade I & $3(13.0)$ & $29.7(26-34)$ \\
$\quad$ Grade II & $9(39.1)$ & $30.8(24-40)$ \\
$\quad$ Varicocele lII & $11(47.9)$ & $32.7(24-41)$ \\
$\quad$ Bilateral & & \\
$\quad$ Unilateral & $15(65.2)$ & $32.5(24-39)$ \\
Testicular volume $\left(\mathrm{cm}^{3}\right)$ & $8(34.8)$ & $30.7(26-38)$ \\
$\quad$ Normal & & \\
$\quad$ Atrophic & $21(91.4)$ & $31.2(24-41)$ \\
$\quad$ & $2(8.6)$ & $38.0(34-40)$ \\
\hline
\end{tabular}

and external spermatic perforators were isolated and divided. An operating microscope (Opmi Visu 160, Zeiss) was then brought into the operating field, and the cord was examined under 8- to 15 -power magnification. Once the internal and external spermatic fascias were incised, the underlying internal spermatic artery and external spermatic artery were identified by their subtle pulsations. The arteries were then dissected free from the underlying veins and encircled with a 2-0 silk suture for identification. Care was taken to preserve lymphatics to prevent the development of postoperative hydrocele and lymphocele. All internal spermatic veins with the exception of the vasal veins were then ligated with 4-0 silk and divided. Then, the operation was ended. Although the diagnostic open testicular biopsy has been recommended at the same time as varicocele repair, it was not performed in order not to extend the operation time.

In postoperative evaluation, semen analyses were obtained at 3 and 6 months after the varicocele repair. The laboratory technician had no knowledge of the diagnosis. The most-improved postoperative semen analysis was used for data analysis.

\section{Results}

The mean age of the patients included in our study was $31.7 \pm 7.4$ years. The mean follow-up was 11.4 months (range 3-18 months). The varicocele was grade I in 3 patients (13.0\%), grade II in 9 patients $(39.1 \%)$, and grade III in 11 patients $(47.9 \%)$ (table 1$)$. The mean testicular volume of patients was $16.2 \pm 3.6 \mathrm{~cm}^{3}$ for the left testis, and $14.3 \pm 4.1 \mathrm{~cm}^{3}$ for the right testis. Of the 23 patients, 14 had normal serum FSH $(7.41 \pm 3.5 \mathrm{ng} / \mathrm{ml})$ and 7 had high levels of serum FSH $(19.2 \pm 6.9 \mathrm{ng} / \mathrm{ml})$. In all patients, serum testosterone levels were normal. The mean preoperative FSH level was $13.05 \pm 4.9 \mathrm{ng} / \mathrm{ml}$ 
Table 2. Semen analysis after microsurgical subinginal varicocele repair and varicocele grade of patients

\begin{tabular}{lllll}
\hline & Grade I & Grade II & Grade III & Total \\
\hline Presence of sperm $(\mathrm{n})$ & 1 & 2 & 4 & 7 \\
Mean sperm concentration $(\times 10 \% \mathrm{ml})$ & 0.001 & 1.3 & 2.9 & 3.34 \\
Mean sperm motility $(\%)$ & 35 & 42.5 & 35.75 & $37.5 \pm 15.5$ \\
Pregnancy rate $(\mathrm{n})$ & 0 & 1 & 2 & 3 \\
\hline
\end{tabular}

(range $1.5-10 \mathrm{ng} / \mathrm{ml}$ ) and the testosterone level was 3.6 $\pm 2.4 \mathrm{pg} / \mathrm{ml}$ (range $8-55 \mathrm{pg} / \mathrm{ml}$ ). There was no significant difference between the preoperative and postoperative serum parameters in all patients.

Bilateral varicocelectomy was performed on 15 patients and unilateral (left) varicocelectomy was performed on 8 patients. In the postoperative period, of the 23 patients, 7 (30.4\%) had motile sperm in the ejaculate. The mean sperm concentration was $1.34 \pm 2.6 \times 10^{6} / \mathrm{ml}$. The mean total sperm motility of these patients was 37.5 $\pm 15.5 \%$.

After subinguinal microsurgical varicocelectomy, sperm was detected in the ejaculate in 1 of 3 patients (33.3\%) with grade I varicocele, in 2 of 9 patients (22.2\%) with grade II varicocele and in 4 of 11 patients $(36.3 \%)$ with grade III varicocele (table 2).

There were no intra- or post-operative complications in any patient. Hydrocele formation, wound infection and recurrence of varicocele (checked by physical examination and scrotal ultrasound) were not observed in any patient during follow-up. After the surgery, one patient achieved spontaneous pregnancy and two patients achieved pregnancy with ICSI in follow-up. These pregnancies include live births.

\section{Discussion}

Varicocele is a dilatation of the pampiniform plexus, which drains blood from the testes. Normally, reverse blood flow is prevented by small valves. Defects in these valves or compression of the veins by adjacent structures can cause vessel dilatation. The effects of varicocele, caused by several mechanisms, are varied but often can result in progressive deterioration and generalized im- pairment of sperm production characterized by abnormal semen quality, ranging from oligospermia to complete azoospermia [1, 12]. Azoospermia is defined as the complete absence of sperm in the ejaculate. Azoospermia is an extreme testicular dysfunction that occurs in patients with varicocele and is a significant barrier to the male factor contribution to unassisted pregnancy. The prevalence of varicocele in men with azoospermia is varied and is estimated to range from 4 to15\% $[5,8,15]$. In recent years, the relationship between varicocele and azospermia has been investigated. We also investigated the relationship between varicocele and azospermia and evaluated the effect of microsurgical varicocelectomy in men with azoospermia.

Varicocele repair by occlusion of the affected spermatic veins can result in improvements in scrotal discomfort, semen parameters and spontaneous pregnancy [16]. Repair is performed surgically or by embolization. Approaches to surgical varicocelectomy are retroperitoneal, inguinal, subinguinal or laparoscopic [17]. The primary benefit of varicocele repair in men with non-obstructive azoospermia is the possibility of producing motile sperm in the ejaculate. When a choice is available, using motile sperm from fresh ejaculate is preferable to TESE in preparation for ICSI [18]. Fresh ejaculated sperm have superior ICSI success rates compared with sperm retrieved by TESE. In addition to providing a sample of greater quality, an invasive and potentially damaging procedure is avoided [19]. In our study, we treated 23 patients with non-obstructive complete azoospermia surgically with the microsurgical subinguinal approach. There were no intra- and post-operative complications. After varicocele repair, we detected motile sperm in the ejaculate of 7 of the 23 patients with non-obstructive complete azoospermia $(30.4 \%)$. 
Although the effect of varicocelectomy has been well studied in oligospermic men, the benefit of this surgical procedure in those with non-obstructive azoospermia has been limited and controversial [20,21]. With the several techniques used (microsurgical or conventional), studies on complete azoospermic men have shown consistent improvement in semen parameters in 22 to $50 \%$ of the cases and even in spontaneous pregnancy [5, 8, 22-24].

The results of our study and the previous studies in which microsurgical subinguinal techniques for varicocele repair was used are similar. In the study of Matthews et al. [8], it was reported that 12 of the 22 patients $(54.5 \%)$ had motile sperm in the ejaculate after subinguinal microsurgical varicocelectomy, with a $27.7 \%$ spontaneous pregnancy rate. In another study, Schlegel et al. [6] reported that 7 of the 31 patients $(22.5 \%)$ had motile sperm in the ejaculate after varicocelectomy, with a $3.2 \%$ spontaneous pregnancy rate. In our study, we also used the microsurgical subinguinal lymph- and arterial-sparing approach for ligation of internal spermatic veins. Of the 23 azoospermic patients, 7 (30.4\%) showed motile sperms in their ejaculate postoperatively.

In several studies using different varicocele repair techniques, similar results were obtained by microsurgical subinguinal technique [11]. In a study by Kadioglu et al. [5], the importance of sperm pellet analysis was evaluated, and it was reported that pellet (+) azoospermic patients had significantly different characteristics when compared to pellet (-) azoospermic cases. They reported that the improvement of semen parameters in men with pellet (-) azoospermia were $20.8 \%$ and men with pellet $(+)$ were $85.7 \%$. In our study, pellet (-) azoospermic (complete azospermic cases) patients were included and the improvement of semen parameters in these patients was $30.4 \%$.

Testicular histology is considered to be a significant predictive factor and has been reported to be an important predictive factor of outcome $[8,11]$. Patients with germ cell aplasia and maturation arrest at the spermatocyte stage did not improve semen quality while azoospermic men with improved semen quality postoperatively are among those with maturation arrest at the spermatid stage and hypospermatogenesis $[8,11,12]$. We did not perform testicular biopsy simultaneously with varicocele repair. Open testicular biopsy was performed on patients who had no motile sperm in the ejaculate after varicocele repair and who had motile sperm but achieved no spontaneous pregnancy. Although it may be a weakness of our study, we maintain that a biopsy has no contribution to improvement of semen parameters after varicocele

Microsurgical Varicocelectomy in Men with Non-Obstructive Azoospermia repair. Biopsy may be useful in predicting the outcome of varicocele repair in azoospermic patients when performed before varicocelectomy.

In conclusion, the results of our study demonstrate that men with non-obstructive azoospermia may benefit from varicocele repair, resulting in motile sperm in the postoperative ejaculate. Motile sperm were found in the postoperative ejaculate in $30.4 \%$ of our patients with nonobstructive azoospermia, resulting in pregnancy in $13 \%$ (in 3 patients of all 23). The present study suggests that varicocele repair can be considered for men with non-obstructive azoospermia who have a varicocele. Subinguinal microsurgical lymph-sparing varicocelectomy can be considered as an optional treatment modality in selected non-obstructive azoospermic patients with varicocele. However, the couple should be counseled that assisted reproductive techniques will mostly likely be required to initiate a pregnancy. Additional studies with more patients and longer follow-up to determine the benefit of varicocelectomy for men with non-obstructive azoospermia are needed.

\section{Acknowledgements}

In this study, one-third of men with complete azoospermia benefited from microsurgical varicocelectomy. Motile sperm in the ejaculate was detected after microsurgical varicocele repair. 


\section{References}

$>1$ World Health Organization: The influence of varicocele on parameters of fertility in a large group of men presenting to infertility clinics. Fertil Steril 1992;57:1289-1293.

$>2$ Stephen EH, Chandra A: Declining estimates of infertility in the United States: 1982-2002. Fertil Steril 2006;86:516-523.

$\checkmark 3$ Dohle GR, Colpi GM, Hargreave TB, Papp GK, Jungwirth A, Weidner W; EAU Working Group on Male Infertility: EAU guidelines on male infertility. Eur Urol 2005;48:703-711.

4 Czaplicki M, Bablok L, Janczewski Z: Varicocelectomy in patients with azoospermia. Arch Androl 1979;3:51-55.

$>5$ Kadioglu A, Tefekli A, Cayan S, Kandirali E, Erdemir F, Tellaloglu S: Microsurgical inguinal varicocele repair in azoospermic men. Urology 2001;57:328-333.

6 Schlegel PN, Kaufmann J: Role of varicocelectomy in men with nonobstructive azoospermia. Fertil Steril 2004;81:1585-1588.

7 Cakan M, Altug U: Induction of spermatogenesis by inguinal varicocele repair in azoospermic men. Arch Androl 2004;50:145150.

$>8$ Matthews GJ, Matthews ED, Goldstein M: Induction of spermatogenesis and achievement of pregnancy after microsurgical varicocelectomy in men with azoospermia and severe oligoasthenospermia. Fertil Steril 1998;70:71-75.

>9 Su LM, Palermo GD, Goldstein M, Veeck LL, Rosenwaks Z, Schlegel PN: Testicular sperm extraction with intracytoplasmic sperm injection for nonobstructive azoospermia: testicular histology can predict success of sperm retrieval. J Urol 1999;161:112-116.
10 Youssef T, Abd-Elaal E, Gaballah G, Elhanbly S, Eldosoky E: Varicocelectomy in men with nonobstructive azoospermia: is it beneficial? Int J Surg 2009; 7:356-360.

11 Weedin JW, Khera M, Lipshultz LI: Varicocele repair in patients with nonobstructive azoospermia: a meta-analysis J Urol 2010; 183: z2309-2315.

12 Gorelick JI, Goldstein M: Loss of fertility in men with varicocele. Fertil Steril 1993;59: 613-616.

13 World Health Organization: Laboratory Manual for the Examination of Human Semen and Sperm-Cervical Mucus Interaction. ed 4. New York, Cambridge, 1999, p3.

14 Goldstein M, Gilbert BR, Dicker AP, Dwosh $\mathrm{J}$, Gnecco C: Microsurgical inguinal varicocelectomy with delivery of the testis: an artery and lymphatic sparing technique. J Urol 1992;148:1808-1811.

15 Kim ED, Leibman BB, Grinblat DM, Lipshultz LI: Varicocele repair improves semen parameters in azoospermic men with spermatogenic failure. J Urol 1992;162:737740.

6 Pryor JL, Howards SS: Varicocele. Urol Clin North Am 1987;14:499-513.

17 Cayan S, Shavakhabov S, Kadioglu A: Treatment of palpable varicocele in infertile men: a meta-analysis to define the best technique. $\mathbf{J}$ Androl 2009;30:33-40.
18 Palermo G, Joris H, Devroey P, Van Steirteghem AC: Pregnancies after intracytoplasmic injection of single spermatozoon into an oocyte. Lancet 1992;340:17-18.

19 Aboulghar MA, Mansour RT, Serour GI, Fahmy I, Kamal A, Tawab NA, Amin YM: Fertilization and pregnancy rates after intracytoplasmic sperm injection using ejaculate semen and surgically retrieved sperm. Fertil Steril 1997;68:108-111.

20 Witt MA, Lipshultz LI: Varicocele: a progressive or static lesion? Urology 1993;42:541543.

21 Kibar Y, Seckin B, Erduran D: The effects of subinguinal varicocelectomy on Kruger morphology and semen parameters. J Urol 2002; 168:1071-1074.

22 Esteves SC, Glina S: Recovery of spermatogenesis after microsurgical varicocele repair in azoospermic men is related to testicular pathology. Int Braz J Urol 2005;31:541-548.

23 Ishikawa T, Kondo Y, Yamaguchi K Sakamoto Y, Fujisawa M: Effect of varicocelectomy on patients with unobstructive azoospermia and severe oligospermia. BJU Int 2007; 101:216-268.

24 Gat Y, Bachar GN, Everaert K, Levinger U, Gornish M: Induction of spermatogenesis in azoospermic men after internal spermatic vein embolization for the treatment of varicocele. Hum Reprod 2005;20:1013-1017. 